\title{
Energy induced rheology reduction of flocculated thickened tailings - pipeline system design methodology
}

\author{
J.M. Treinen Paterson \& Cooke Ltd, United States of America \\ R. Cooke Paterson \& Cooke Ltd, United States of America \\ C. Salinas Paterson \& Cooke Consulting Engineers (Pty) Ltd, Chile
}

\begin{abstract}
Salinas et al. (2009) demonstrated the relationship between energy input and rheology reduction for flocculated thickened copper tailings. This phenomenon has important implications for the design of thickened and paste tailings pipeline and open channel systems. This paper presents the development of a bench top test to determine the energy input versus rheology reduction characteristic curve. The results of the bench top test are used to establish a design methodology for incorporating the rheology reduction into the design of paste and thickened tailings systems.
\end{abstract}

\section{Introduction}

The floc-aggregate structures of flocculated mineral slurries are altered by shearing the mixture resulting in a reduction in the mixture rheology. The rheological term for this phenomenon is rheomalaxis; an irreversible decay in rheology when a mixture is exposed to shear. While some flocculated mineral slurries exhibit partial recovery after being left quiescent for some time, this decay can generally be treated as irreversible within the time frame of interest for engineering paste and thickened tailings systems.

Salinas et al. (2009) reported the results of an investigation into this effect, their primary findings were:

- The rheology of flocculated copper tailings can be significantly reduced by shearing the mixture.

- A number of shear devices were tested with the finding that the rheology reduction is a function of the energy introduced into the mixture and is independent of the device configuration.

- The rheology reduction for a given slurry, concentration and flocculant dosage can be correlated with the energy input per unit volume.

This paper expands on this work by detailing a bench top viscometer test procedure to characterise the rheology reduction, highlighting the complications and considerations in developing the procedure, and discussing how the rheology reduction can be taken into consideration when designing thickened and paste tailings pipeline and open channel systems.

\section{Rheology reduction measurement}

\subsection{Measurement procedure}

Salinas et al. (2009) detailed an in-line shear device and procedure developed to evaluate the rheology reduction of flocculated tailings. Paterson \& Cooke has developed a methodology for evaluating rheology reduction in a laboratory environment using a rotational viscometer. The testing is completed using a cup and rotor configuration; for the results presented here a Haake Viscotster 550 viscometer with a MV-II cup and rotor configuration has been used. A constant shear test is conducted, where the rotor is rotated at a constant speed and the resulting torque on the rotor is measured. The test is run until the measured torque, which decreases as the floc-aggregate structure is broken and the rheology decreases, reaches a stable, 'fully sheared' level.

The testing presented here was conducted at 133 RPM, which corresponded to a shear rate at the rotor of $120 \mathrm{~s}^{-1}$. Each constant shear test was run for 15 minutes to ensure that the sample was fully sheared. Figure 1 
presents typical data measured for a constant shear test. Once the sample is fully sheared, it is then possible to run a standard flow curve test, where the wall shear stress is measured over a range of 0 to 333 RPM $\left(300 \mathrm{~s}^{-1}\right.$ shear rate at the rotor). If the sample is fully sheared, the results from ramping up from 0-333 RPM should match those on the ramp back down to 0 . If not, it indicates that the sample may not be fully sheared, and still exhibits rheomalaxis.

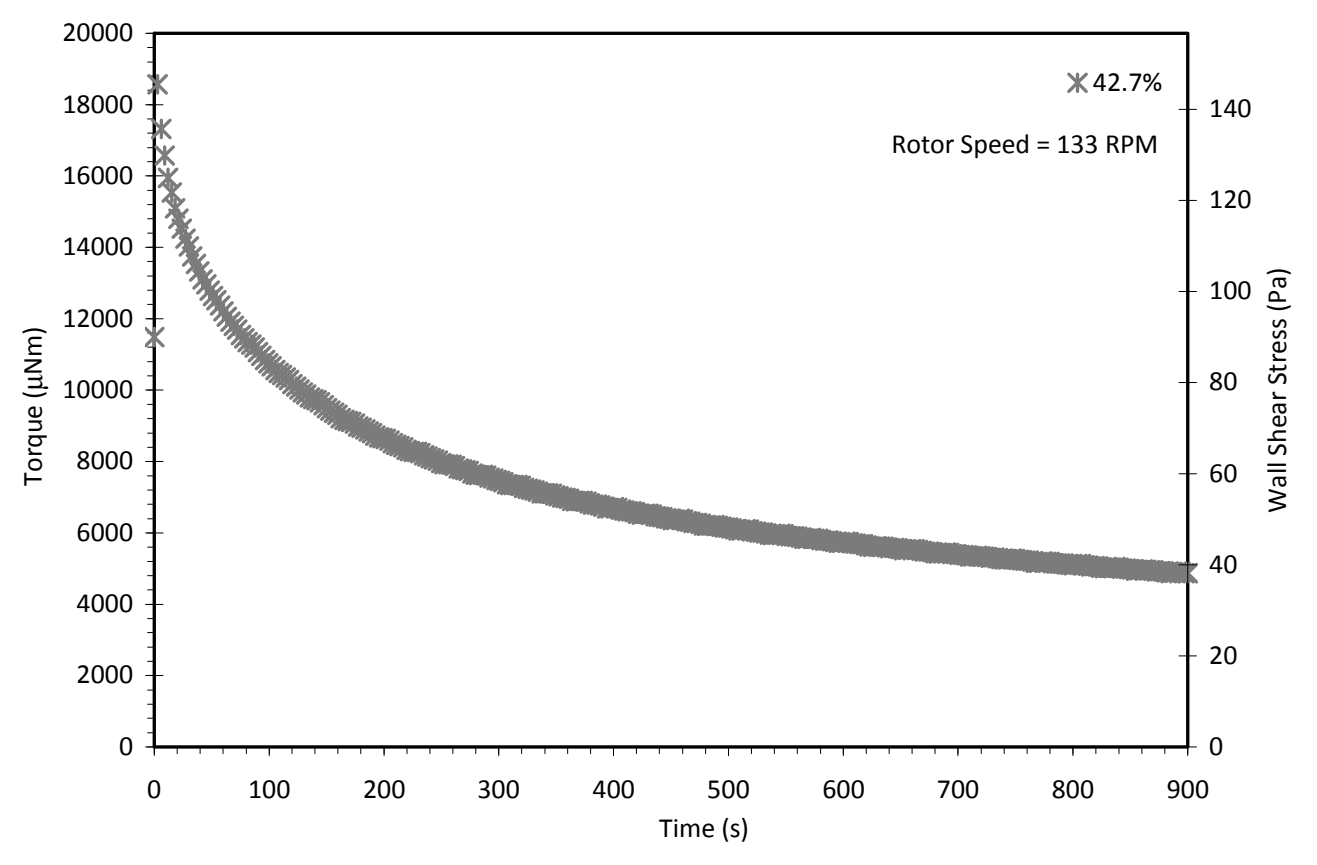

Figure 1 Typical results from a constant shear test

\subsection{Measurement challenges}

\subsubsection{Particle size}

One problem in using a rotational viscometer to evaluate the shear reduction effect is that particle settling tends to occur in tailings samples with large sized particles. Typically, to fully shear a sample requires testing over 10 to 15 minutes, in this time the large particles may settle at the bottom, resulting in an artificially lower fully sheared wall shear stress. Figure 2 presents an example of this type of behaviour for coarse copper tailings, where the fully sheared wall shear stress is measured to be $15 \%$ lower than what is measured using a typical flow curve measurement on a fully sheared, homogenously mixed sample. This can be compared to the results of fine potash tailings slurry shown in Figure 3, where the constant shear and flow curve results correspond well.

Only the finer particles (typically considered to be finer than $20 \mu \mathrm{m}$ ) participate in the flocculation process. Sieving the coarse particles out of the slurry prior to flocculation may alleviate the settling problem and produce shear reduction behaviour representative of the complete sample. Further investigation is ongoing to validate the applicability of this proposed methodology. 


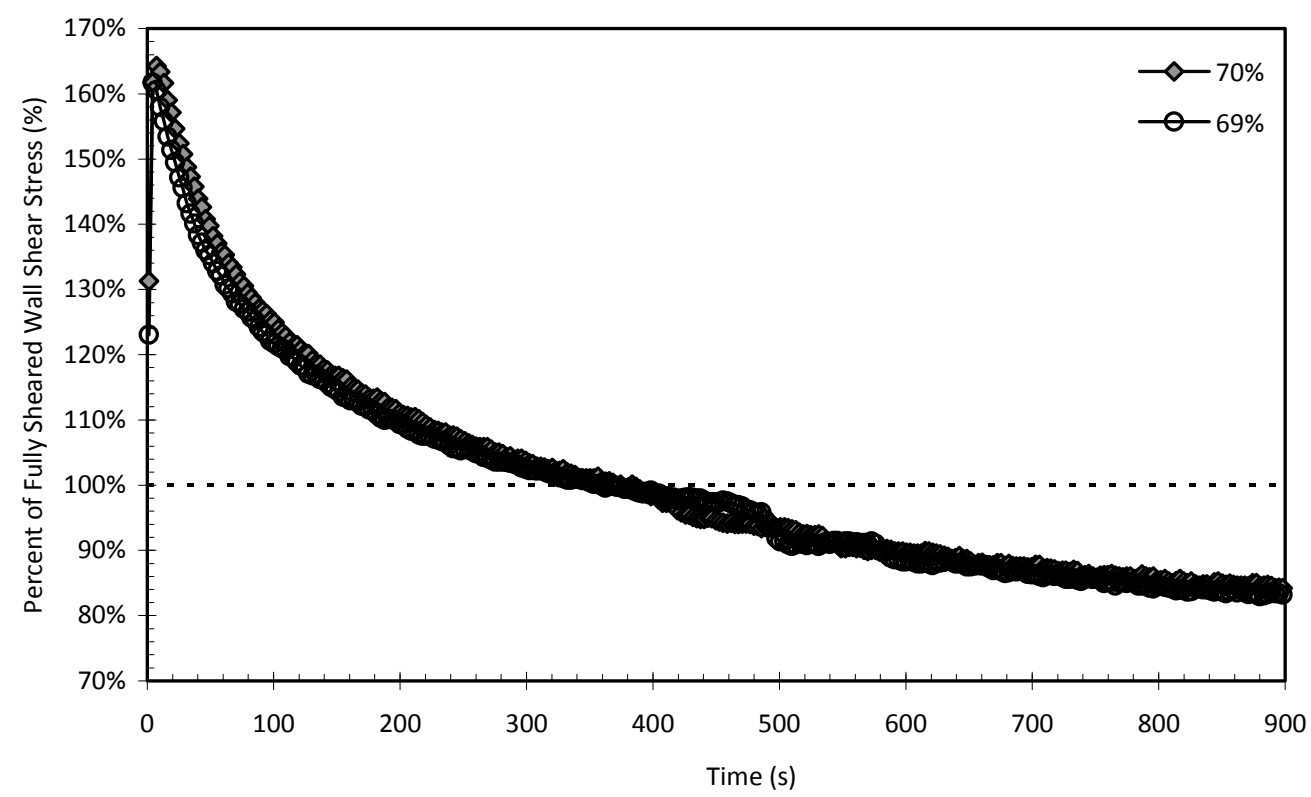

Figure 2 Coarse copper tailings slurry with settling during testing

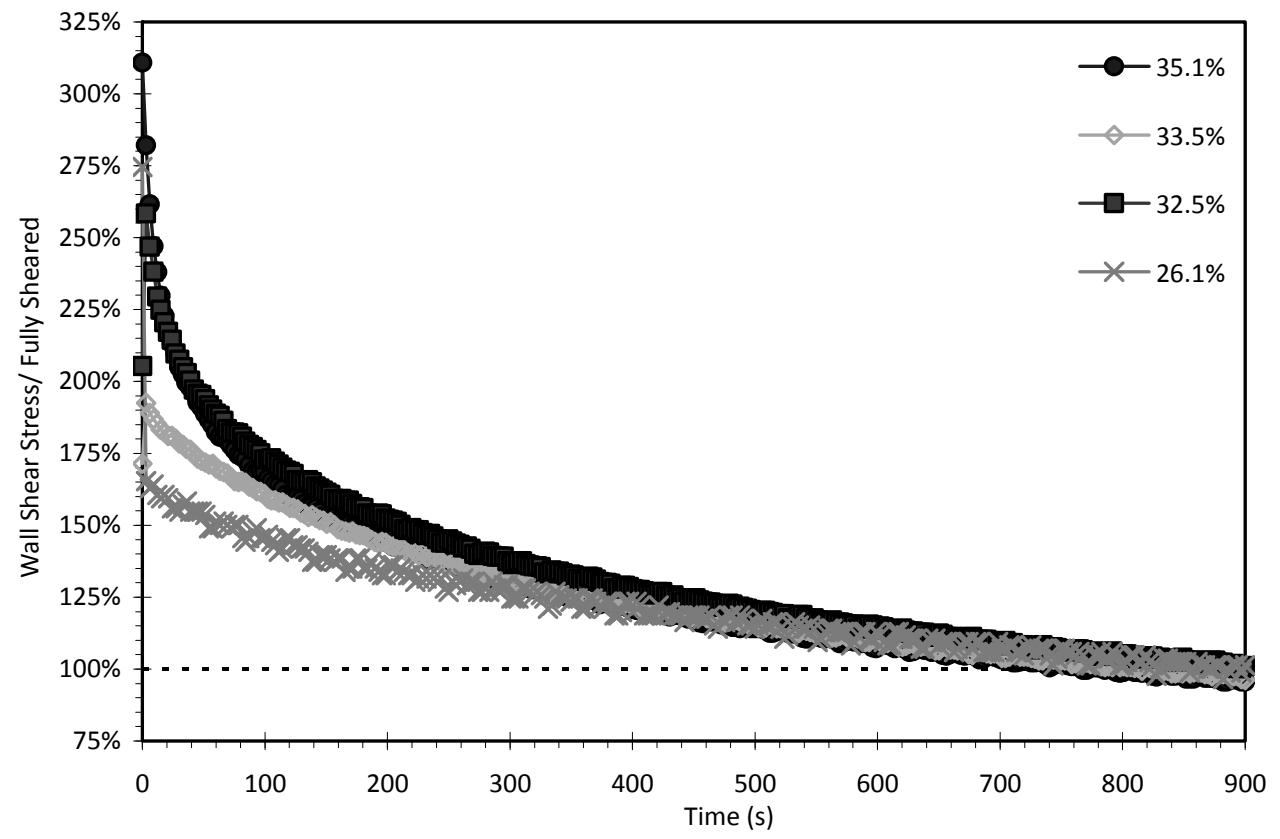

Figure 3 Fine potash tailings with negligible settling during testing

\subsubsection{Pre-test rheology reduction}

Another challenge in rotational viscometer testing is that the actual completely unsheared rheology cannot be measured. Shear reduction, to some degree, will always occur as the sample is transferred from the bench-top thickener or container where it has been thickened into the rotational viscometer. The authors currently assume that this inadvertent reduction in rheology is fairly negligible as long as the sample is not significantly disturbed prior to testing.

Additionally, flocculent decays over time, so constant shear tests should be completed immediately after thickening. If the testing is done even a few days after thickening, the rheology reduction may appear to be 
less than will be seen in a continuous plant operation and the system design may not appropriately take this into consideration.

\section{Data analysis}

With the constant shear data recorded, it is first necessary to develop a relationship correlating the reduction in wall shear stress with the absorbed energy per unit volume of slurry mixture. The advantage of calculating the specific absorbed energy per volume is that the calculations can be scaled to larger volumetric pipe flows. As with the Salinas et al. (2009) paper, the absorbed energy can be calculated based on the torque and speed from the rotational viscometer, and the time step interval between measurements:

$$
\mathrm{E}=\frac{\text { Power } \cdot \text { time }}{\text { Volume }}=\frac{\mathrm{T} \omega}{\pi\left(\mathrm{R}_{1}{ }^{2}-\mathrm{R}_{2}{ }^{2}\right) \mathrm{L}} \cdot \Delta \mathrm{t}
$$

where:

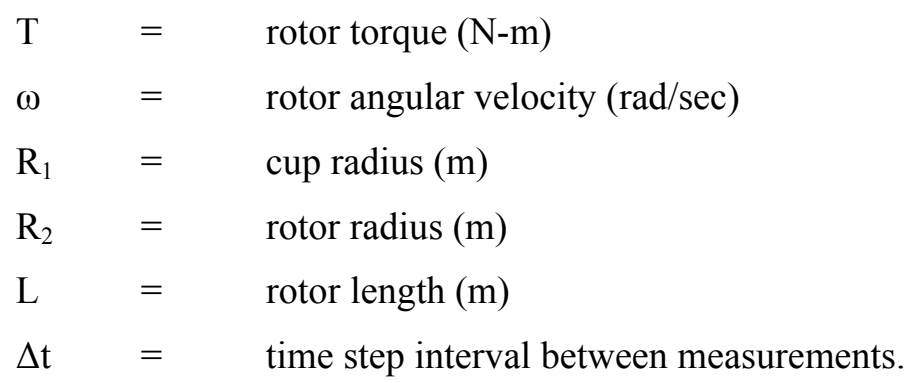

The summation of the absorbed energy for each data point provides the total energy absorption at each time step in the test.

This method for predicting rheology reduction in pipe flow from rotational viscometer tests was validated by comparing the rheology reduction in the rotational viscometer to that measured in pilot plant work using the shear device in the Salinas et al. (2009) paper; Figure 4 presents the comparison. The results from the pilot plant and the viscometer correlate well.

The ratio of the wall shear stress and the fully sheared wall shear stress measured from the flow curve test can then be plotted as a function of the absorbed energy. It is possible to fit an empirical equation to the test data of the form:

$$
\frac{\tau_{w}}{\tau_{w F S}}=f(\mathrm{E})
$$

where:

$\tau_{\mathrm{w}} / \tau_{\mathrm{wFS}}=\quad$ ratio of wall shear stress to full sheared stress

$\mathrm{E} \quad=$ absorbed energy.

The actual correlation function is selected to best suit the specific tailings rheology reduction characteristic. Generally, exponential or power law type decay functions are appropriate. Figure 5 presents test data for potash tailings at two different mass concentrations plotted as a function of absorbed energy. 


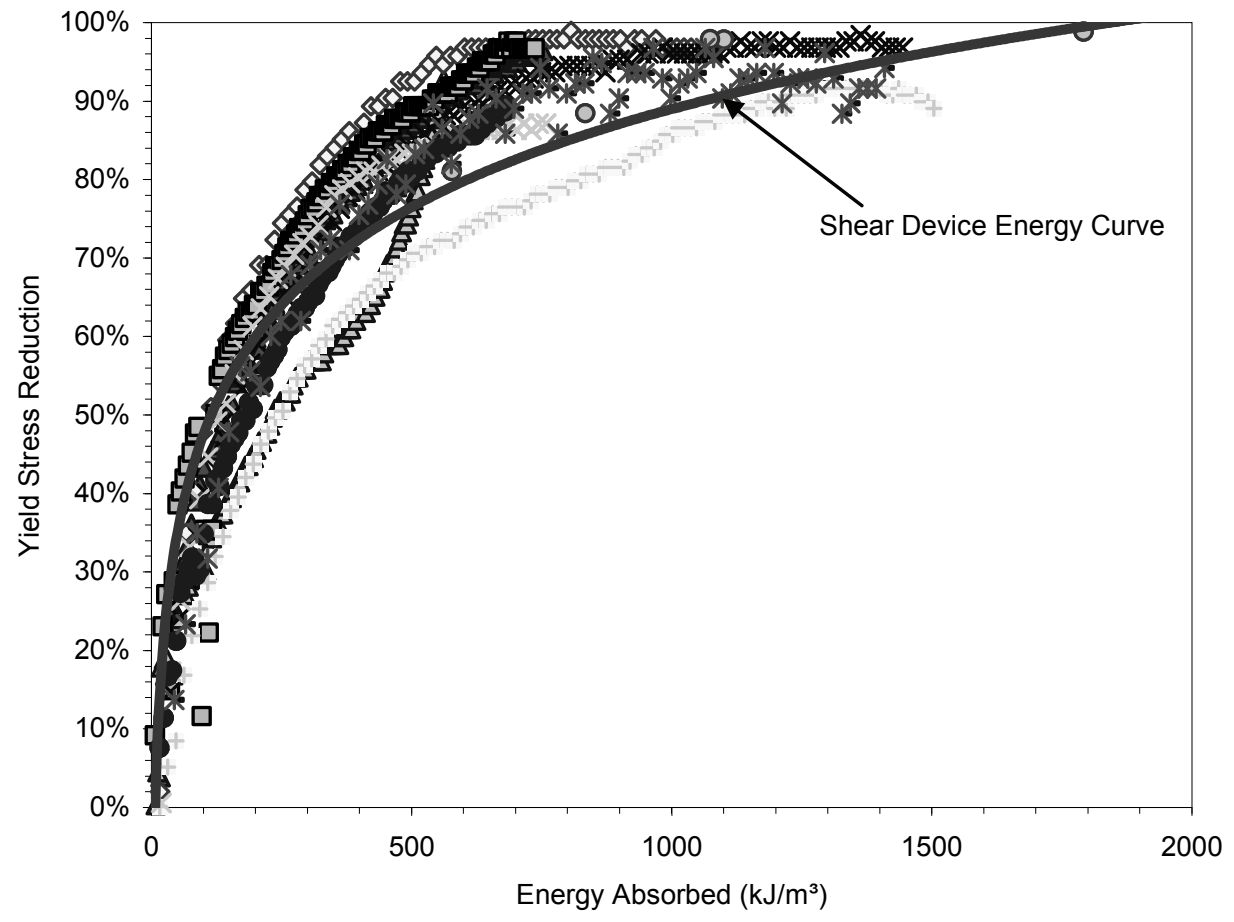

Figure 4 Comparison of rotational viscometer (points) and pilot plant shear device (solid line)

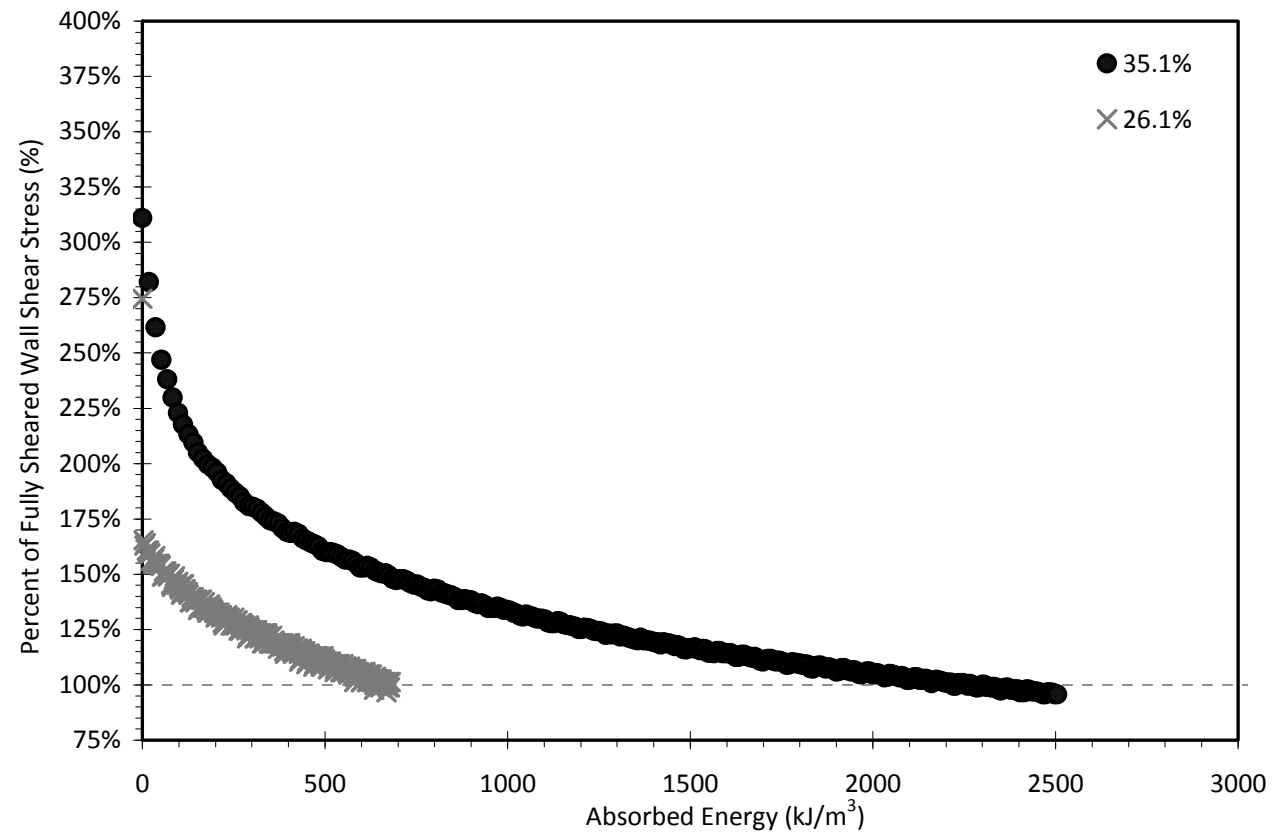

Figure 5 Shear reduction as a function of absorbed energy, including correlations

\subsection{Concentration dependency}

The work of Salinas et al. (2009) indicated that the shear reduction per unit of volume of the underflow was similar over a small range of concentrations. Further work over a much broader concentration range indicates that the energy input required to achieve fully sheared rheology is concentration dependent. Figure 6 shows a power law relationship between the energy required to reach $100 \%$ of the fully sheared rheology and the 
concentration. The scatter in the data is potentially due to the differences in pre-testing shearing to which each sample is subjected.

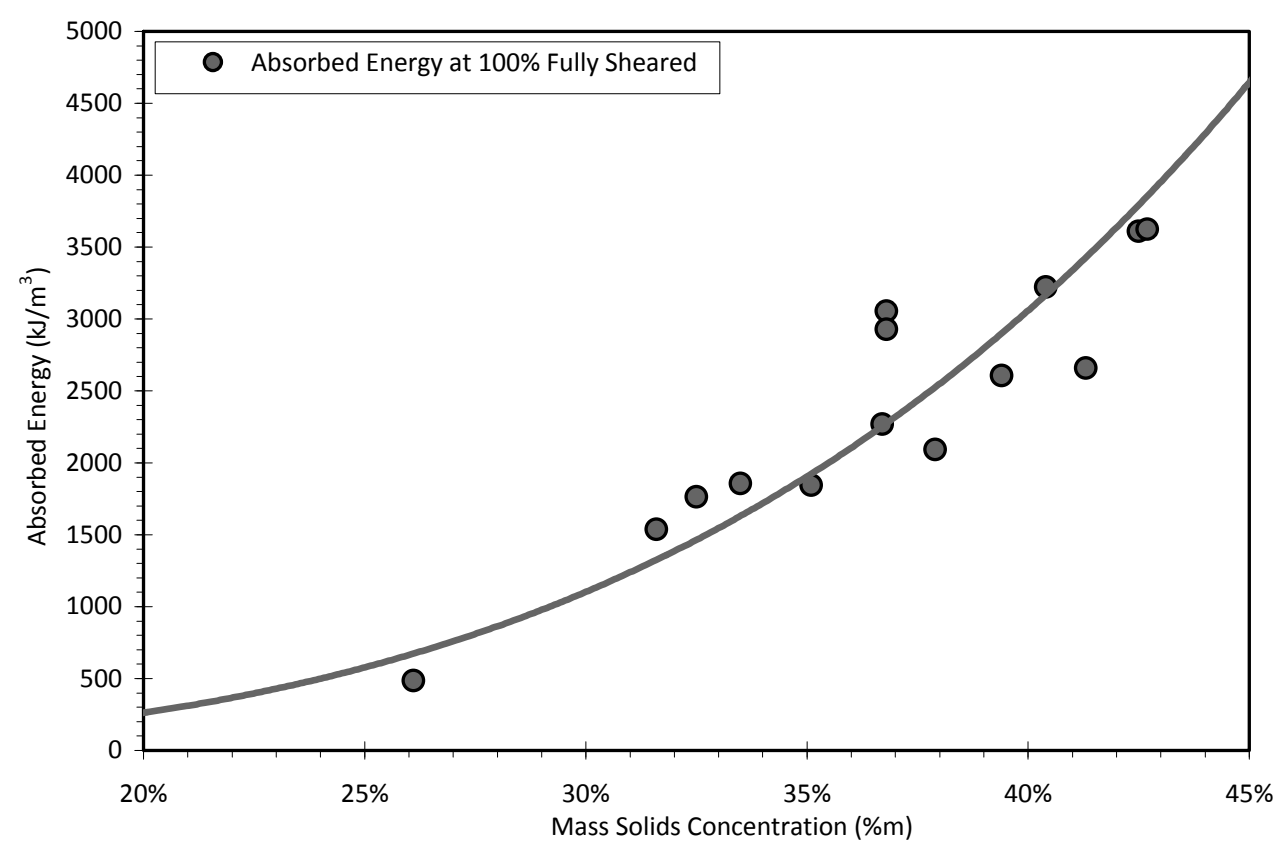

Figure 6 Absorbed energy to achieve $100 \%$ fully sheared versus solids concentration

\section{System design methodology}

\subsection{Assumptions}

The following assumptions are made in developing the design methodology:

- The rheology reduction for a given slurry, concentration and flocculant dosage is correlated with the energy input per unit volume. It is assumed that the rheology reduction is independent of energy input mechanism as demonstrated by Salinas et al. (2009).

- The methodology assumes that the measured constant shear data accurately depicts the unsheared behaviour, i.e. that there is minimal pre-test shearing.

- The mixture yield stress decreases proportionally to the wall shear stress measured during the constant shear test, i.e. it is assumed that the Bingham plastic viscosity remains constant, and that shearing only results in a decrease in yield stress.

\subsection{Design procedure}

The following procedure can be used in the hydraulic design of underflow systems to take into account the reduction in rheology:

1. Discretise the piping system into pipe elements. Shorter element lengths increase the simulation accuracy and it is suggested that short elements $(1 \mathrm{~m}$ length) are used for the start of the piping system, but these can be increased up to $100 \mathrm{~m}$ for end of the pipeline. Some iteration is required to optimise the selection of element lengths.

2. Starting at the thickener outlet, calculate the pipeline friction losses in the first pipe length, using the unsheared yield stress. The unsheared yield stress is found using Equation (2) at zero absorbed energy.

The amount of energy absorbed by the slurry over that length can then be calculated based on the pipe friction losses and the following equation: 


$$
\mathrm{E}_{\text {absPipe }}=\frac{d P}{d x} Q \Delta L
$$

where:

$\mathrm{dP} / \mathrm{dx}=\quad$ friction hydraulic gradient calculated based on the rheology for that element

$\mathrm{Q}=$ volumetric flow rate

$\mathrm{dL}=$ pipe element length.

Using this absorbed energy, the reduced rheology for the next segment of pipe can then be predicted.

1. The friction losses and absorbed energy can then be calculated for that segment. This procedure continues for the entire length of piping. Figure 7 provides a schematic of the calculation procedure.

2. For pumps in the system, the actual pumping head, and consequently the pumping efficiency is unknown until the friction losses are known for the entire system. It is necessary to assume no rheology reduction caused by the inefficiency of the pump (in reality the pump may be the primary source of rheology reduction, particularly in short systems) and calculate the friction losses for the entire system. The pumping head can then be calculated, and the efficiency determined based on the duty point.

3. The pump energy absorbed by the slurry can then be included in the rheology reduction calculations as shown in Figure 8, and the procedure repeated, now considering the pump energy, to more accurately predict the hydraulic grade line for the system. Iterating several times will produce an accurate representation of the rheology reduction, and subsequent friction losses, in the system. The resulting hydraulic grade line for the system will be non-linear.

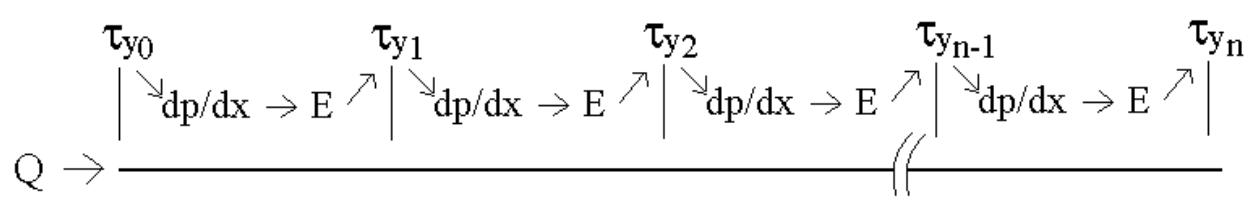

Figure 7 Initial rheology reduction calculation along pipe/launder length

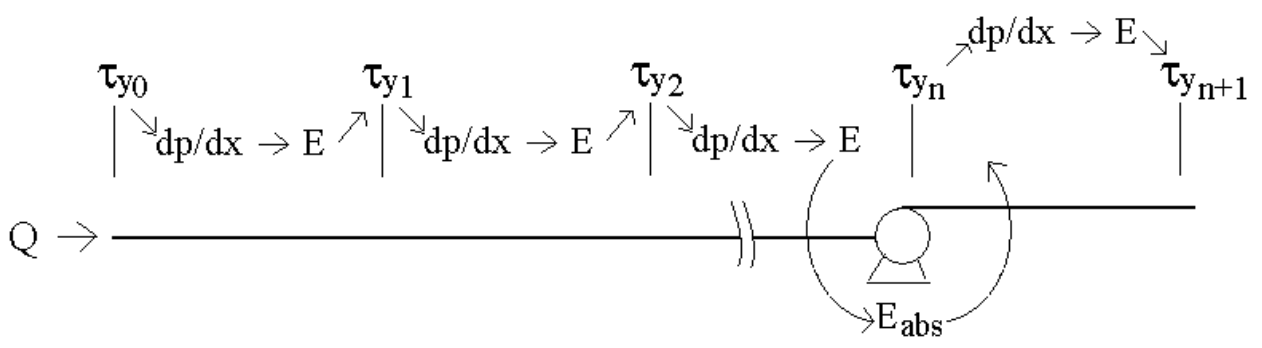

Figure 8 Rheology reduction including shear reduction due to pump inefficiency

\section{Conclusions}

The shearing of floc-aggregate bonds significantly reduces the rheology of paste and thickened tailings. A bench top laboratory procedure using a rotational viscometer for characterising the reduction has been presented. Coarse particle settling, pre-test sample preparation, and shear energy consideration are discussed. The paper details a design methodology developed by Paterson \& Cooke for modelling the effect of rheology reduction in paste and thickened tailings systems. Application of this methodology more accurately represents the performance of systems transporting flocculated tailings. 


\section{References}

Salinas, C., Martinson, R., Cooke, R. and Ferrada, O. (2009) Shear and Rheology Reduction for Flocculated Thickened Tailings, in Proceedings of the Twelfth International Seminar on Paste and Thickened Tailings, Vina Del Mar, Chile April, 2009, Australian Centre for Geomechanics, Perth, Australia, pp. 3-10. 